\title{
Design of Modern Elevator Group Control Systems
}

\author{
Tsung-Che Chiang and Li-Chen $\mathrm{Fu}$ \\ Department of Computer Science and Information Engineering \\ National Taiwan University, Taipei, Taiwan, R.O.C. \\ \{r88008, lichen\}@csie.ntu.edu.tw
}

\begin{abstract}
To provide good transportation services for passengers in the modern buildings, a good elevator group control system (EGCS) is inevitably necessary. The viewpoint of designing the EGCS is very important. Passenger-based viewpoint proposed here provides a new way to think about this system. The capacity constraint following the thoughtfulness consideration for the passengers is utilized to make the performance better. Details of elevator dynamics are modeled to meet the requirements. A traffic database is constructed in order to rebuild the system environment containing information of passengers so that it can be made as close to the real environment as possible. The rescheduling ability is achieved by the new mechanism - HCPM refinement, which is a priority maker for hall calls.

The advantages of our EGCS are shown through extensive simulation results. We make a comparison between our EGCS and the previous one, which shows that our results are quite satisfactory and superior.
\end{abstract}

\section{I . INTRODUCTION}

There are more and more high-rise buildings in the cities for the spatial and economical considerations. To provide transportation services among floors, elevator systems are installed as primary service facilities. Elevator group control system (EGCS) is responsible for controlling elevators to provide convenient and comfortable services for passengers.

What the EGCS wants to solve is a resource allocation problem in a changing environment. It should coordinate multiple elevators and dispatch elevators to serve call requests efficiently based on any information it can collect. Each time a new request comes, the EGCS should evaluate possible solutions and choose an appropriate elevator to serve it. The algorithm used to make decisions and the evaluation criteria are very important to the effectiveness of the EGCS.

In order to obtain accurate evaluation results, elevator dynamics is inevitably the first key point. Contract speed and acceleration determine time needed to move between floors and affect service time. In this paper, we describe elevator dynamics in detail.
Most EGCS try to cut waiting time and riding time of calls as much as possible but ignore the fact that it is passengers whom actually need to be satisfied. We stand from the passengers' viewpoint and this strategy really gives better performance.

In many cases, traffic in a building follows a temporal routine. A good EGCS can collect traffic data, identify traffic patterns and adapt itself to various traffic conditions. By recording call information and passenger information, we can build a traffic database whereby future traffic prediction and passenger-based evaluation can thus be achieved.

Since traffic is always changing, after a call request arises, an elevator is dispatched to serve the request but may be found unsuitable to do so after a period right before the request is actually served. The EGCS should take care of this condition and choose another appropriate elevator according to new status of the system. The ability to perform dynamic scheduling, which is the salient feature of our series of researches, is greatly improved here. A mechanism used to position the idling elevators is also proposed in this paper.

The remainder of this paper is organized as follows. In Section II we list the major considerations in designing an EGCS. Section III gives the details about how we design our EGCS. Analysis on simulation performance is shown in Section IV. Finally we have a conclusion in Section V.

\section{II . MAJOR CONSIDERATIONS OF DESIGNING AN EGCS}

Many researchers have worked in this field for a long period. Each research group has their own interests. In this section, we want to list what we think to be the major considerations of designing an EGCS.

\section{A. Elevator Dynamics}

Elevator dynamics include everything related to elevators. Contract speed and acceleration are important to estimate waiting time and riding time. Moving time can be calculated from floor to floor or from position to position. Loading/unloading actions can be modeled in 
different ways.

\section{B. Evaluation Criterion}

Evaluation criteria usually can be translated into the objectives which the EGCS tries to achieve. Reducing waiting time is commonly the most important objective of each EGCS.

\section{Evaluation Methods}

To evaluate the performance indices, many different methods have been used. Simulation method [1] completes this work by elaborate computations. Fuzzy expert systems $[2,3]$ match system status to each fuzzy rule and then combine the consequents to predict performance. Artificial neural network [4] should be trained and then learn the relationship between the system status and the performance. Besides the above methods, these indices can also be calculated by mathematical formula.

\section{Hall Call Assignment Method (HCAM)}

Hall call assignment method is an algorithm which utilizes the powerful computation ability brought by computers to dispatch elevators to serve hall calls efficiently. Each time a new hall call comes, there are generally three steps in HCAM:

(1) Input the current traffic condition

(2) Performance predictions

(3) Determining the service elevator

\section{E. Traffic Adaptability}

Knowing current traffic condition is very helpful to evaluate possible assignments and then make a good schedule. A good EGCS can even send elevators to floors before the hall call request occurs. Collecting traffic data can predict the occurrence of hall calls and car calls, and use traffic data to plan the routes of elevators to give more efficient services.

\section{F. Rescheduling Ability}

Although the EGCS selects an appropriate elevator when a new hall call comes, this assignment may become unsuitable because of changes of system status. Therefore, a good EGCS should adjust the previous assignment according to the most current system situation. An EGCS able to do this is said to have the rescheduling ability. Relatively few EGCS's have this ability in the literature.

\section{DESIGN OF OUR EGCS}

Fig. 1 gives the overview of the entire elevator system. In this section we will present how we build the EGCS, which is the lower block in this figure. First we describe the elevator dynamics in the system. Then how we collect traffic data will be explained. HCAM and the related virtual elevator system are the next. Finally we explore hall call priority maker (HCPM) and the control policy unit.

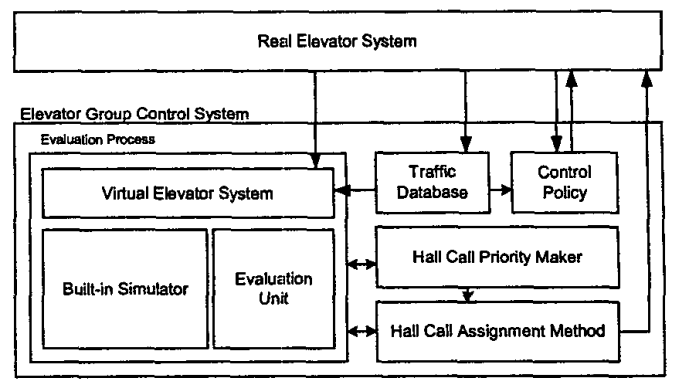

Fig. 1 The overview of the elevator system.

\section{A. Elevator Dynamics}

We use three modules to manage the behaviors of elevators.

\section{Basic Movement Module}

The contract speed and acceleration are two important parameters to describe the movement of the elevator. Moving time for an elevator to reach the destination floor is calculated with the above two parameters and its current speed and the distance. It is calculated from position to position instead of from floor to floor. This model is more practical and the obtained moving time is more accurate.

\section{Call Management Module}

In our model, call management module builds a stop list for each elevator. Hall calls and car calls assigned to an elevator are arranged in order, and the elevator moves and serves calls in this order.

When a call is assigned to an elevator, CM module inserts it into an appropriate position in the stop list. Generally, up/down hall calls which arise at lower positions have higher/lower service priority than those which arise at higher positions. If the elevator is not moving, the first hall call assigned to it determines the moving direction of this elevator.

Each time when an elevator starts to move or the stop list is modified, the elevator looks up its stop list and the $\mathrm{BM}$ module determines how it moves to serve the first call in the list.

Besides building the stop lists for elevators, CM module has other three tasks. It must clear the stop list at the first step of the rescheduling procedure. And it is responsible for sending a command call to a moving elevator with empty stop list after rescheduling, so that the elevator can stop safely at the nearest floor. The last duty is sending the express call to make idling elevators stop at the floors with high passenger arrival rate. 


\section{Loading/Unloading Module}

When an elevator arrives at its destination floor, it starts to serve a call request. In our model, we assume that riding passengers will walk out of the elevator before waiting passengers go into the elevator. Another assumption that is related to $\mathrm{CM}$ module is that riding passengers will press car call buttons right away after they get into the elevator.

In our model, time for loading and unloading one passenger is a constant value. Total servicing time is proportional to the number of waiting passengers and riding passengers. With this model, the possibility that a new hall call is assigned to an elevator earlier dispatched to serve a floor with many passengers is reduced. Hence, system performance can be improved.

Since elevators in our system serve for passenger not for calls, capacity issue is considered here. Elevators have contract capacity, and a full elevator cannot load any more passengers. Those left waiting passengers will press the hall call button right after the elevator leaves.

\section{B. Traffic Database}

Traffic information in the building came into researchers' mind in the recent years. It was collected to do performance prediction, to tune parameters in the evaluation functions, and to adjust the operation policies for elevators. Here, we take two parameters to represent the traffic condition - passenger arrival rate and car call probability on each floor. Passenger arrival process is assumed to be a Poisson Arrival Process, as what it is generally assumed.

In order to estimate the passenger arrival rate and car call probability, we assume that each elevator is equipped with a weight sensor which can measure weights of riding passengers. Collected data is recorded in a traffic database. We define the length of a time interval as $T$, and each time interval has its own record for passenger arrival rate $r_{a r r}$ and a record for car call probability $p_{c c}$. The element $r_{a r r}[i]$ is the estimated average passenger arrival rate of the floor $i$. The element $p_{c c}[j][i]$ is the estimated probability that a waiting passenger on the floor $i$ wants to go to floor $j . w$ is a scalar which means the amount of weight changes in the elevator. $W$ is a fixed value used to estimate the number of passengers, which is set 60 here without loss of generality. The 1-D array, wpnum, records the number of passengers from each floor. cctable is 2-D array, and the estimated number of passengers coming from floor $i$ will be recorded in $c c t a b l e[j][i]$ for all registered car call $j$.

\section{Estimation process}

All elements in wpnum and cctable are initialized to zero at the beginning of a time interval.

\section{Passenger Arrival Rate}

Each time when an elevator serves a hall call on the floor $i$, the weight sensor measures the amount of increased weight, $w$. The ratio $w / W$ is added in wpnum[i]. At the end of the time interval, wpnum[i]/T is taken as the average passenger arrival rate.

$$
r_{a r}[i]=\frac{w p n u m[i]}{T}
$$

\section{Car Call Probability}

Each time when an elevator serves a hall call on the floor $i$, the weight sensor measures the amount of increased weight, $w$. For all floors $j$ whose corresponding car calls are registered at that time, the ratio $w / W$ is recorded in all cctable $[j][i]$.

Each time an elevator serves a car call on the floor $j$, the weight sensor measures the amount of decreased weight, $w$. Let $L$ be $w / W$. If there is only one non-zero element in some $i_{\mathrm{th}}$ column of the array cctable, then

$$
\begin{aligned}
& p_{c c}[j][i]=p_{c c}[j][i]+\min \{c c t a b l e[j][i], L\} \\
& L=L-\min \{c c t a b l e[j][i], L\}
\end{aligned}
$$

Once $L$ becomes 0 , this process terminates. If $L$ is still positive after doing the equation (2) for each column as what we mentioned above, the value $L$ is shared by all floors $i$ where cctable[j][i] is not zero in proportion to the value of cctable[j][i]. These values will be added to $p_{c c}[j][i]$.

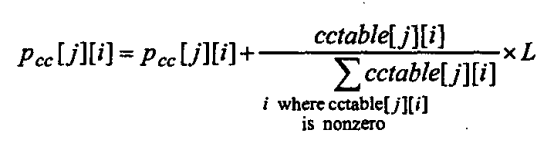

And then for these floors $i$, the shared number $S$ (the second term at the right side of equation (3)) of $p_{c c}[j][i]$ is subtracted from all other nonzero elements cctable $[k][i]$ except cctable[j][i]. cctable[j][i] is set zero.

$$
\begin{aligned}
& \text { cctable }[k][i]=c c t a b l e[k][i]-S \forall \text { cctable }[k][i] \text { nonzero and } k \neq j \\
& \text { cctable }[j][i]=0
\end{aligned}
$$

At the end of the time interval, the car call probability for a passenger from floor $i$ to go to floor $j$ is calculated by

$$
p_{c c}[j][i]=\frac{p_{c c}[j][i]}{\sum_{j} p_{c c}[j][i]}
$$

The general idea of the above operations is sharing leaving riding passengers by the number of passengers coming from each floor. And the car call probability from each floor is estimated by the data of its own shared numbers.

\section{Virtual Elevator System and HCAM}

The hall call assignment method is responsible for 
assigning hall calls to elevators so as to provide passengers good transportation services. The HCAM in our EGCS calculates two cost values for each assignment. One is calculated before the new hall call is assigned to an elevator, and the other is obtained after the call is assigned. And it selects the elevator that has the smallest difference value. It is the same as we proposed in [1].

Three evaluation criteria are considered in our evaluation process - waiting time, riding time and power consumption. The significant difference from the other existing researches is that waiting time and riding time are evaluated for each passenger rather than for each call. In this subsection we will show how we model passengers in two aspects: waiting passengers and riding passengers.

\section{Waiting passengers}

To create data concerning waiting passengers, we must take care of three properties for each passenger, namely, arrival time, destination floor, and weight. Weight will be taken to justify whether the elevator is overloaded. Weight of each waiting passenger is simply made as 60 . When waiting passengers enter the elevator, their arrival time is used to calculate the waiting time. If a waiting passenger cannot be served because the elevator is full, his waiting time will be evaluated in the way of RWT, as we will mention in the end of this subsection. Creation of arrival time and destination floor resorts to the traffic database to complete.

\section{Creation process}

Passengers are created by the Poisson arrival process on each floor. If there is an unserviced hall call, the process starts from the time when the last hall call was registered. Otherwise, this process starts from the current system time. The average passenger arrival rate for each floor is taken from the traffic database.

Once a passenger is created, his destination is determined by the car call probability recorded in the traffic database.

\section{Riding passengers}

For riding passengers, their destination floors are the first property to be made. It determines where these riding passengers leave and to what degree these passengers will affect hall calls assigned to the elevator. To calculate riding time for riding passengers, we need to know the time when they went into the elevator. Passengers' weight is made in the same way as how we do for waiting passengers.

\section{Creation process}

We estimate the number of riding passengers first. It can be achieved by the data from the weight sensor. If the number is less than the number of car calls, it is set as the number of car calls, and then passengers' weight are set as the ratio of total weight to the number of passengers.

The 2-D array cctable in the traffic table now comes in. Riding passengers are shared by those floors whose corresponding columns have nonzero elements. For example, if cctable[3][2] is 3 and cctable[3][4] is 4, passengers' source floors will be generated to be floor 2 and 4 with probability $3 / 7$ and $4 / 7$.

After determining each passenger's source floor, his loading time is set as the time when the elevator lastly stops at the source floor. Since a passenger's source floor is known, his destination floor can be generated by the car call probability table $P_{c c}$ recorded in the traffic database.

\section{Evaluation}

When the virtual elevator system is available, we can use this environment to do simulation to evaluate possible assignments and obtain cost values in HCAM. Four evaluation items used are listed as follows.

- Passenger's waiting time (WT)

- Failed-loading passenger's waiting time (RWT)

- Passenger's riding time (RT)

- Elevator moving distance (PC)

Here waiting time and riding time are evaluated with each passenger instead of each call as the unit. This point of view to evaluate is very helpful to make a good schedule. For example, we will provide better service for the floor with more waiting passengers than the floor with only few waiting. passengers. We can also use the destination information of riding passengers to plan the service routes. Failed-loading passenger's waiting time is evaluated when the elevator is full and some passengers can not be loaded. With this evaluation criterion involved, our EGCS can utilize the capacity information of elevators. Thus, our EGCS can prevent a nearly full elevator from stopping redundantly. It is very helpful to reduce waiting time and riding time during down-peak traffic pattern.

Above four evaluation items are combined in the form of weighted sum,

$$
\text { Cost }=w_{1} \sum_{\substack{\text { i:waitng } \\ \text { passengers }}}\left(W T_{i}^{2}+R W T_{i}^{2}\right)+w_{2} \sum_{\substack{\text { iriding } \\ \text { passengers }}} R T_{i}^{2}+w_{3} P C
$$

where $w_{1}$ to $w_{3}$ are tuned according to the importance of each item.

\section{D. $H C P M$}

In most EGCS's, a hall call will never be reassigned after it was assigned to an elevator. As the system status like positions of elevators and the distribution of call requests changed, the previous assignment made by the EGCS may be unsuitable and may cause bad effect on the dispatching result for new coming hall calls. Therefore 
rescheduling is necessary. Here we propose a priority-based mechanism which reschedules hall calls without evaluating all possible assignments. This mechanism arranges the order of hall calls to do HCAM.

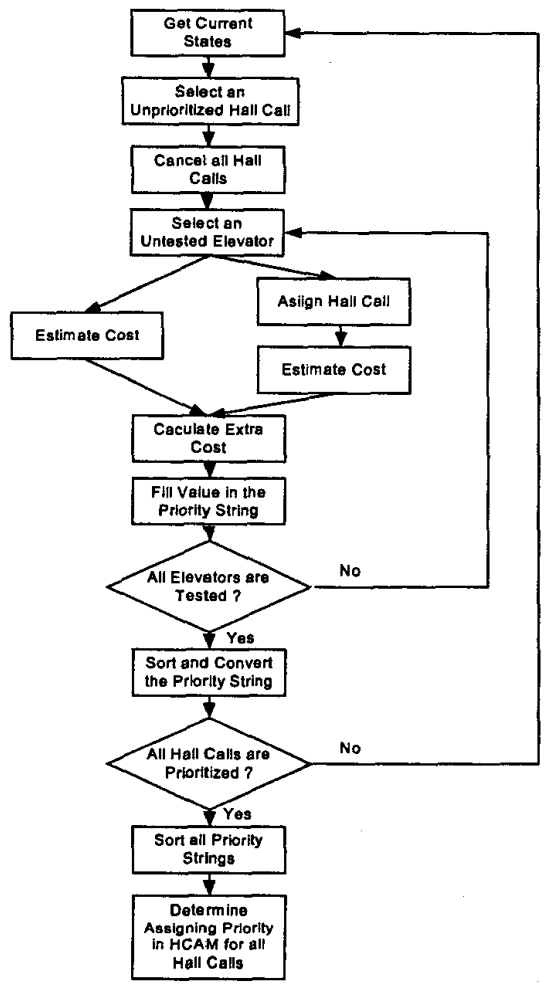

Fig. 2 Flow of hall call priority maker (HCPM)

\section{HCPM Process (Fig. 2)}

1) Pick a hall call $h c$ whose priority string is not made.

1.1) Cancel all previous assignment except hall calls that are serving.

1.2) Set all elevators untested. Prepare a 1-D array pstr with size as the number of elevators for the hall call $h c$. Set a variable $i=0$.

1.3) Pick an untested elevator ele and calculate the $\cos c_{l}$ in the same way we used in the HCAM.

1.4) Assign the hall call $h c$ to the elevator ele, and calculate the cost $c_{2}$.

1.5) Fill $p s t r[i]$ with $c_{2}-c_{1}$, and set $i=i+1$. If there is any elevator untested, return to Step 1.3; otherwise, go to Step2.

2) Sort the array pstr in the increasing order.

3) Convert the array pstr by $p \operatorname{str}[i]=p s t r[i+1]-$ pstr[i]. If there is any hall call whose priority string is not made, return to Step1; otherwise goto Step 4.

4) Sort these priority strings and give hall calls priority based on the sorting result.

\section{Meaning of HCPM}

For each hall call, all elevators are tested to determine the suitability of them by the extra cost, i.e. $c_{2}-c_{1}$ in Step1.5 in the HCPM process. These values are recorded in the priority string of the hall call. Sorting the elements in the priority string arranges the suitability values from the best one to the worst one. And then after converting the priority string, each element pstr $[i]$ in the string has the meaning that how much the hall call will get lost if it is assigned to the $(i+1)_{\text {th }}$ best elevator instead of the $(i)_{\mathrm{th}}$ best one. At the end of HCPM, each converted priority string are compared to decide which hall call should be assigned earlier, or in other words, which hall call should have chance to occupy its favorite elevator than others.

\section{E. Control Policy}

If we can predict where passengers will come, we can park the idling elevator at those floors and reduce their waiting time. The control policy unit in our EGCS is responsible for managing the idling elevators. Here we will explain how it works.

\section{Operation Policy Process}

Traffic records in the database used to express current traffic situation change with time. Each time the record changes, we will sort floors with their arrival rates and calculate how many idling elevators are deserved for them. It is calculated by

IdlingElevatorDeserved $[i]=\frac{\operatorname{rate}[i]}{\sum_{j} \text { rate }[j]} \times E L E N U M$

As for those floors whose arrival rates are not large enough to keep one idling elevator, we will integrate them as several sections. If the sum of arrival rates of the floors in a section is large enough, the middle floor of this section will deserve an idling elevator. This is useful to park idling elevators separately when the inter-floor traffic dominates. Then, when an elevator $j$ serves all its calls and becomes an idling elevator, we do the following management. (Fig. 3)

1) Do rescheduling of all hall calls. If the elevator $j$ becomes busy, this procedure terminates, otherwise, go to Step 2.

2) Select a untested floor $i$ with the largest arrival rate.

3) Find the number of idling elevators already sent to or park at this floor $E_{b}$. If IdlingElevatorDeserved[i] $\geq E_{b}+1$, send the elevator $j$ to the floor $i$.

4) If all floors are tested, terminate this procedure; otherwise, go to Step 2.

The concept of our operation policy is to share idling elevators to the floors by the traffic load. When an elevator becomes idle, rescheduling is invoked to see if it 
is helpful to relieve other elevators' load. If other elevators do not need its participation, we send this elevator to the floor where passenger arrival rate is high.

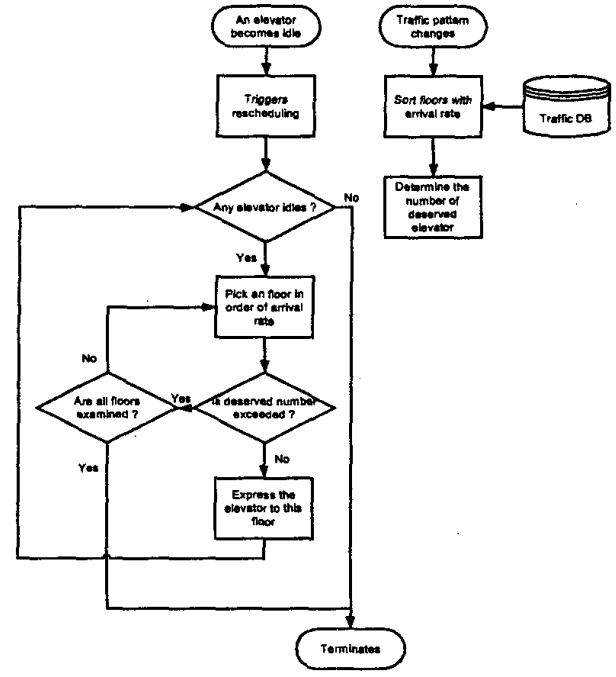

Fig. 3 Flow of control policy

\section{SIMULATION RESULTS AND ANALYSIS}

Here we compare the performance of our EGCS with the previously proposed one by simulation. The building in the simulation model has 15 floors and 4 elevators. Four traffic patterns are examined. Table 1 summarized the major differences between these two systems. And Table 2 gives the comparison of performance. Average waiting time and riding time of passengers are shown.

In the up-peak and down-peak traffic pattern, our new EGCS has very good performance. Under these two patterns, much more information is obtained from employing the passenger-based viewpoint to analyze the elevator system. As for the inter-floor pattern, the improvement primarily comes from the new rescheduling mechanism. And the last one - two-way traffic pattern, there is no significant improvement because of the light traffic load.

Table 1: Major differences between previous and current EGCS

\begin{tabular}{|l|l|l|}
\hline & Previous EGCS & Current EGCS \\
\hline Evaluation Viewpoint & Call-based & Passenger-based \\
\hline Traffic Database & Not used & Used \\
\hline Capacity issue & Not considered & Considered \\
\hline Rescheduling Mechanism & Two-way refinement & HCPM refinement \\
\hline
\end{tabular}

Table 2: Comparison of performance during four traffic patterns

\begin{tabular}{|l|c|c|c|c|c|}
\hline \multicolumn{2}{|c|}{} & Up-peak & Down-peak & Inter-floor & Two-way \\
\hline $\begin{array}{l}\text { Previous } \\
\text { EGCS } \\
\text { (seconds) }\end{array}$ & AWT & 40.2 & 35.4 & 38.5 & 17.6 \\
\cline { 2 - 7 } & ART & 36.6 & 31.5 & 30.0 & 25.7 \\
\hline $\begin{array}{l}\text { Climent } \\
\text { EGCs }\end{array}$ & AWT & 313 & 311 & 36.3 & 17.6 \\
\hline $\begin{array}{l}\text { Impronds) } \\
\text { nt (\%) }\end{array}$ & ART & 36.7 & 24.5 & 29.6 & 23.3 \\
\cline { 2 - 7 } & AWT & 21.6 & 11.0 & 5.7 & 0 \\
\hline
\end{tabular}

\section{CONCLUSION}

In this paper, we proposed a new viewpoint to design the modern EGCS. Thinking from passengers' viewpoint provides much more information to make good schedules for the elevators. This is achieved by construction of temporal traffic database, passenger-based evaluation, and the utilization of the capacity constraints.

We showed how we construct the traffic database by collecting the information available from the real environment. And the method to create a virtual elevator system with passengers' data is also explained here. Dynamics scheduling, which is very important to provide good performance, is done by the priority-based mechanism - HCPM. The control policy manages the positions of idling elevators with the ability to predict future incoming traffic from the traffic database.

The performance of our system is examined with the simulation on four different traffic patterns. The experimental results are very satisfactory.

\section{REFERENCE}

[1] Yuan-Wei Ho and Li-Chen Fu, "Dynamic Scheduling Approach to Group Control of Elevator Systems with Learning Ability," Proceedings. ICRA '00. IEEE International Conference on Robotics and Automation, vol. 3, pp. $2410-2415,2000$.

[2] Chang Bum Kim, Kyoung A. Seong and Hyung Lee-Kwang et al., "Design and Implementation of a Fuzzy Elevator Group Control System," IEEE Transactions on Systems, Man and Cybernetics Part A, vol. 28 , issue 3, pp. $277-287$, May 1998.

[3] T. Ishikawa A. Miyauchi and M. Kaneko, "Supervisory Control for Elevator Group by Using Fuzzy Expert System which also Addresses Traveling Time," Proceedings of IEEE International Conference on Industrial Technology, vol. 2, pp. 87-94, 2000.

[4] Zhu Dewen, Jiang Li, Zhou Yuwen and Shan Guanghui et al., "Modern Elevator Group Supervisory Control Systems and Neural Networks Technique," IEEE International Conference on Intelligent Processing Systems, vol. 1, pp. 528 - 532, 1997. 